\title{
1 Introducing a Cantellation Strategy for the Design of Mesoporous Zeolite-like Metal-Organic Frameworks: Zr-sod-ZMOFs as a Case ${ }_{3}$ Study
}

${ }_{4}$ Norah Alsadun, ${ }^{\S}$ Georges Mouchaham, ${ }^{\S}$ Vincent Guillerm, Justyna Czaban-Józwiak, s Aleksander Shkurenko, Hao Jiang, Prashant M. Bhatt, Prakash Parvatkar, and Mohamed Eddaoudi*

Cite This: https://dx.doi.org/10.1021/jacs.0c10007

Read Online

6 ABSTRACT: Herein we report novel mesoporous zirconium-based metal-organic frameworks (MOFs) with zeolitic sodalite (sod) 7 topology. Zr-sod-ZMOF-1 and -2 are constructed based on a novel cantellation design strategy. Distinctly, organic linkers are 8 judiciously designed in order to promote the deployment of the 12-coordinated $\mathrm{Zr}$ hexanuclear molecular building block (MBB) as a 9 tetrahedral secondary building unit, a prerequisite for zeolite-like nets. The resultant Zr-sod-ZMOFs exhibit mesopores with a 10 diameter up to $\approx 43 \AA$, while the pore volume of $1.98 \mathrm{~cm}^{3} \cdot \mathrm{g}^{-1}$ measured for Zr-sod-ZMOF-1 is the highest reported experimental 11 value for zeolite-like MOFs based on MBBs as tetrahedral nodes.

${ }_{13}^{12} \mathrm{M}$ etal-organic frameworks (MOFs) are a unique class of crystalline materials, resulting from the assembly of 14 organic and inorganic molecular building blocks (MBBs) into 15 periodic structures. ${ }^{1}$ Since the end of the 1990s, tremendous 16 efforts have been dedicated to explore their properties and 17 potential applications. ${ }^{2-4}$

18 Among almost 100000 MOFs reported to date, ${ }^{5}$ the touch 19 of rational design is increasingly visible, thanks to the principles 20 of reticular chemistry and the associated RCSR database. ${ }^{6}$ 21 Indeed, design strategies facilitated the assembly of MOFs with 22 increasing complexity. If the assembly of common MBBs into 23 MOFs based on the reticulation of edge-transitive nets is now 24 widely applied, ${ }^{1 c, 7}$ then researchers are committing noticeable 25 efforts toward expanding the repertoire of reticular chemistry. ${ }^{8}$ 26 This is achievable by using complex ligands to deviate from 27 "default" assemblies" or by developing advanced strategies, 28 such as pillaring ${ }^{10}$ and supermolecular building approaches ${ }^{11,12}$ 29 in order promote the assembly into a precise topology when 30 multiple possibilities are plausible for the same basic building 31 blocks.

32 Interestingly, some of these design strategies are inspired by 33 the well-known porous materials zeolites. These so-called 34 zeolite-like MOFs (ZMOFs) are constructed from tetrahedral 35 building blocks. ${ }^{13}$ Two main ZMOFs design approaches can be 36 distinguished, depending on their basic elements of con37 struction: (i) those based on single-metal ion $\left(\mathrm{Cu}^{2+}, \mathrm{Zn}^{2+}\right.$, $38 \mathrm{Co}^{2+}, \mathrm{In}^{3+}$, etc.) with functionalized imidazole (ZIFs) or 39 imidazole dicarboxylate, leading to diverse structures with 40 known topologies (sod, ana, rho, etc.) ${ }^{14}$ alongside unprece41 dented ones (med) ${ }^{15}$ and (ii) those with corner-sharing of 42 super-tetrahedra constructed from trimeric building blocks and 43 linear (MIL-101) ${ }^{16}$ or triangular ligands (MIL-100), ${ }^{17}$ system44 atically to afford mtn underlying topology. ${ }^{18}$

45 The synthesis of ZMOFs with expanded pores is a persisting 46 challenge. $^{13}$ The common approach to MOFs isoreticular expansion is by adjusting the organic linker, e.g., length and/or 47 width elongation. ${ }^{19}$ However, this strategy is less effective for 48 obtaining highly porous ZMOFs. ${ }^{14 \mathrm{c}}$ This is due to the fact that 49 there are hundreds of zeolitic topologies based on linking 50 tetrahedral building units. Besides, linker expansion/elongation 51 can induce modification in the specific angles and the 52 positioning of the coordinating functional groups, prohibiting 53 the isoreticulation of a specific ZMOF platform. It mostly 54 affords amorphous solids or crystalline materials based on the 55 "default" structure for the assembly of tetrahedral building 56 units, a dia underlying topology. ${ }^{20}$ In this context, the targeted 57 frameworks must meet a specific arrangement of tetrahedral 58 nodes at certain range of angles at the MBB level to reach the 59 desired zeolitic net. As a semiregular edge-transitive net with 60 simple tilling (transitivity 1121), the sodalite (sod) net has the 61 highest occurrence, making it a suitable target for the design of 62 expanded ZMOFs. ${ }^{21}$ Markedly, the reticulation of the sod net 63 with expanded bridging linkers will permit access to open 64 ZMOF structures, due to the fact that the sod net is not self- 65 dual and not prone to self-interpenetration; that is, it has a high 66 pore/aperture size ratio, large pore volume, and contracted 67 pore-aperture size, suitable for molecular entrapment for 68 potential applications. ${ }^{13,14 \mathrm{~b}}$ Herein, we introduce a cantellation 69 design approach for the directed assembly of mesoporous 70 ZMOFs based on zirconium MBBs.

For the rational design a novel generation of isoreticular 72 ZMOFs, we identified three main requirements:

Received: September 18, 2020 
- Achieving the formation of a tetrahedral MBB

- Preventing the formation of dia net, the most regular edge-transitive net, the default topology for the assembly of tetrahedra ${ }^{22}$

- Obtaining an assembly mode that allows the tuning of the framework through ligand expansion and/or decoration

After a careful survey of existing structures and topologies, 82 we reasoned that an efficient method to deviate from the 83 default dia net while assembling tetrahedral nodes would be to 84 propose a solution that prevents their stacking in staggered 85 fashion. For this purpose, we propose a cantellation strategy 86 (Figure 1): Second-order truncation of a tetrahedron, adding a

a

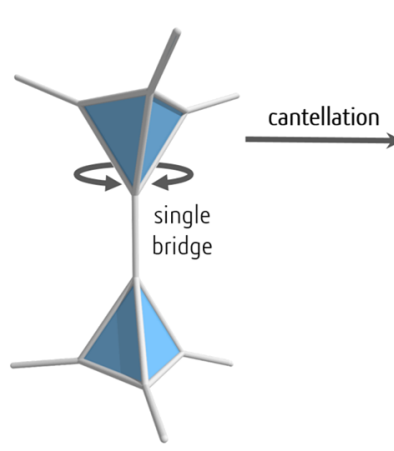

I? free rotation:
favored dia net

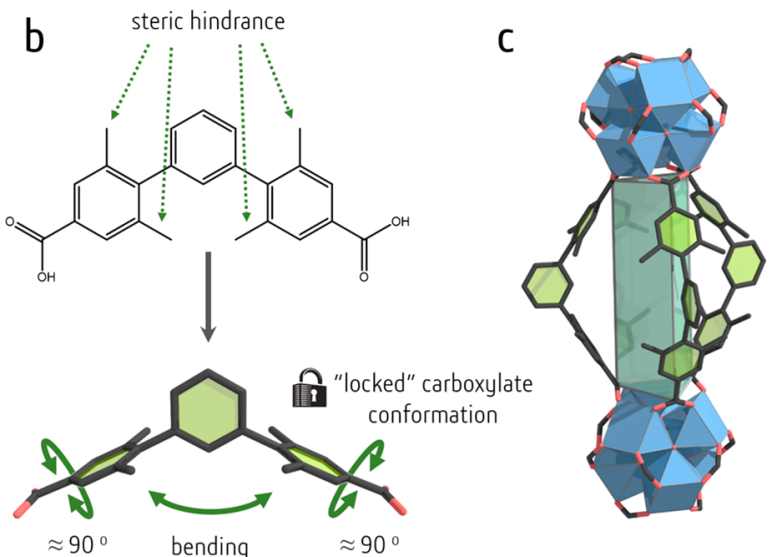

Figure 1. (a) Schematic of the tetrahedra cantellation strategy leading to multiple bridges, allowing building units to be "locked" in eclipsed conformation and (b) sterically hindered bent ligand, locking the carboxylic group in the appropriate position to form (c) a triple bridge between MBBs.

87 face at each edge and vertex of a tetrahedron, affords the 88 formation of cuboctahedron. Applying the same cantellation 89 strategy to a couple of tetrahedra stacked in an eclipsed fashion 90 results in triply bridged cuboctahedra stacked in an eclipsed 91 conformation. At this stage, the triple bridges between the 92 cuboctahedra prevent any rotation and therefore "lock" them 93 in the intended eclipsed fashion.

94 Cuboctahedral (cuo) MBBs are well-known in reticular 95 chemistry, with the ubiquitous $\mathrm{Zr}$ hexanuclear cluster of $\mathrm{UiO}$ $9666{ }^{23}$ Therefore, $\mathrm{Zr}$ represents a natural candidate for further 97 design of a novel class of ZMOFs.
Then, we embarked in the design of an organic ligand that 98 would promote the formation of the necessary triple bridge 99 between the cuo MBBs (Figure $1 \mathrm{~b}, \mathrm{c}$ ). If such an example has 100 been recently reported with linear terephthalic acid in an UiO- 101 66 polymorph with hex-topology, ${ }^{24}$ then this conformation can 102 be regarded as an exception as it is widely accepted that the 103 favored topology would be fcu. ${ }^{23,25} 104$

To deviate from the default fcu topology, one must modify 105 the geometry of the ligand, i.e., introducing a geometry 106 mismatch. ${ }^{9}$ Indeed, systematic explorations have shown that 107 topological diversity can be achieved through the use of bent ${ }^{26} 108$ or zigzag ${ }^{19 c}$ ligands, as well as introducing steric hindrance to 109 alter the orientation of the carboxylates. ${ }^{27}$ To meet our needs, 110 the ideal ligand (i) should be a ditopic linker, with a bent 111 geometry allowing the triple bridging of MBBs without 112 excessive constraint on the Zr-carboxylate bonds, and (ii) 113 have the two carboxylates oriented orthogonally to the plan of 114 the ligand. Separately, these two requirements would not be 115 sufficient. Indeed, controlling only the bending angle on planar 116 ligands afforded wide topological diversity, allowing the 117 formation of MOFs with fcu, ${ }^{28}$ reo, ${ }^{29}$ bon, ${ }^{26} \mathbf{b c t},{ }^{30} \mathbf{h b r},{ }^{31}{ }_{118}$ $\mathbf{k a g}^{32}$ or pcu topologies. ${ }^{33}$ In parallel, introducing steric 119 hindrance on linear ligands favored the assembly of MOFs with 120 bcu topology. ${ }^{27}$

The insightful combination of these two geometrical 122 requirements guided the design and synthesis of benzene- 123 1,3-dimesitylenic acid (BDM, L1, Figure 1b). The ligand is 124 based on a terphenyl core, where the central phenyl group is 125 decorated by two benzoic groups in the meta positions. To 126 fulfill the second criterion, we introduced steric hindrance by 127 the means of methyl groups on the ortho positions (with 128 respect to the central phenyl group) of each of the benzoic 129 groups. In this way, introducing the methyl groups at these 130 specific positions will act as a lock, limiting the rotation of the 131 rings and keeping the peripheral carboxylate groups orthogonal 132 to the plan of the ligand. This overall minimizes the 133 coordination options and favors the triple bridging of the 134 MBBs, virtually reducing their net-connectivity from 12 to 4.135 Each 12-coordinated (12-c) cluster is surrounded by 4 others 136 in a tetrahedral fashion, as a result of the 12 ligands splitting 137 into four triads converging to the same neighboring cluster 138 (Figure 2c).

Reaction of $\mathrm{ZrCl}_{4}$ and $\mathrm{L} 1$ in DMF and acetic acid resulted in 140 isolation of rhombic dodecahedral crystals (Figure S16a). 141 Single-crystal X-ray diffraction studies revealed that the 142 obtained MOF encloses fully coordinated clusters (12-c) and 143 that the overall MOF has the targeted underlying sod 144 topology, observed for the first time in $\mathrm{M}^{\mathrm{IV}}$-based MOFs. 145 Due to the triple bridging of the clusters by the ligands, a 146 resulting cavity is not represented by the classic tiling of the 147 sod topology. It is reflected by the unprecedented augmented 148 net, csd (cantellated sodalite), unveiled thanks to our design 149 approach. It differs from sod-a net, in that it also allows us to 150 appreciate the presence and connectivity mode of the 151 cuboctahedral building blocks, where a trigonal prism 152 completes the tiling (Figures S21-S23).

Zr-sod-ZMOF-1 crystallizes in the $\operatorname{Im} \overline{3} m$ cubic space group 154 with a unit cell parameter of 55.199(7) $\AA$ and a volume of 155 $168190(60) \AA^{3}$. The overall framework contains two types of 156 cages: the large truncated rhombic dodecahedral cage typical 157 from the sodalite net is mesoporous, with a diameter $\approx 43 \AA$, 158 accessible through square windows of $\approx 8 \AA$ and hexagonal 159 windows of $\approx 21 \AA$ apertures. In addition, the structure 160 


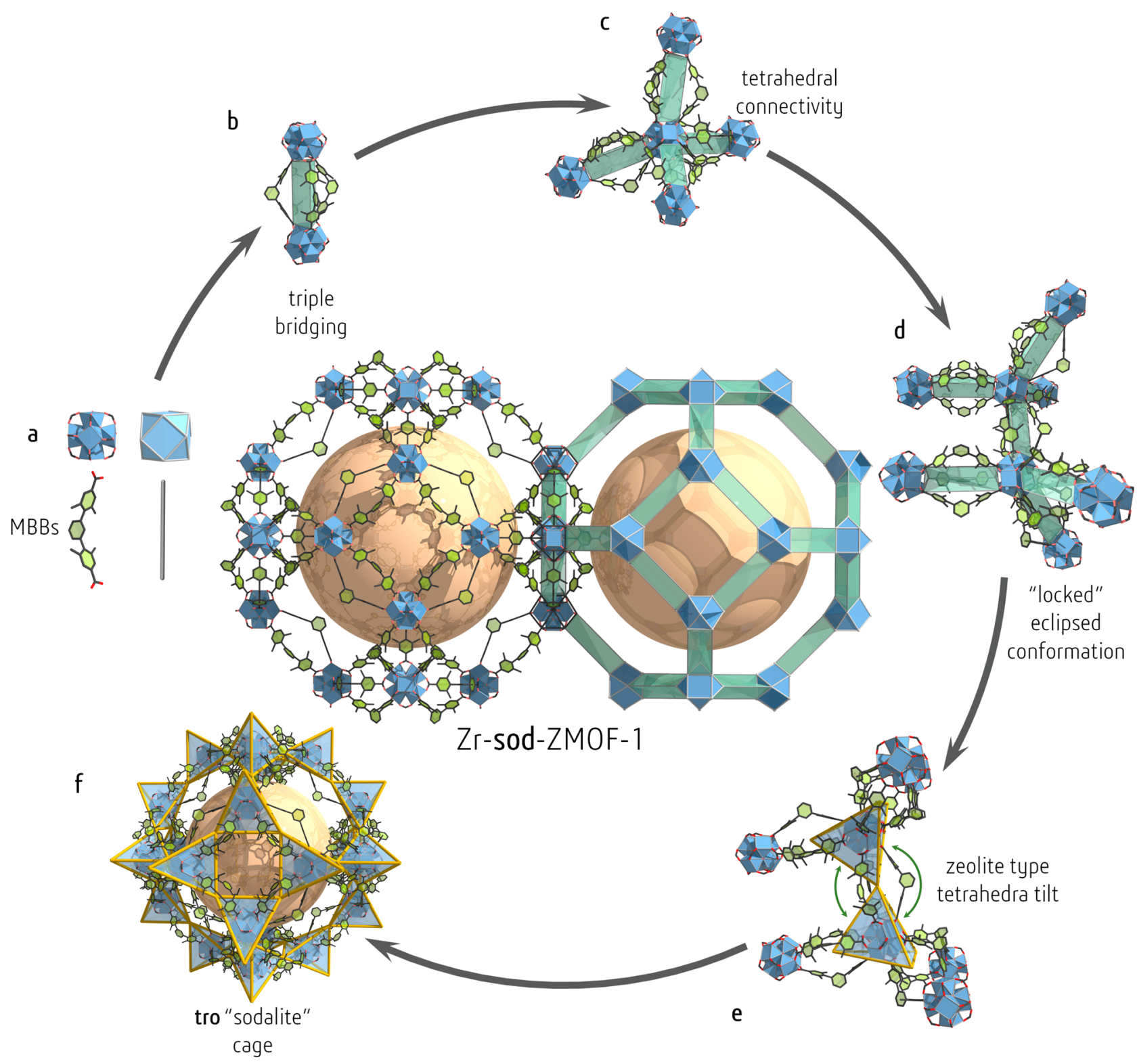

Figure 2. Illustration of the bottom-up construction of Zr-sod-ZMOF-1 (center): (a) Organic and inorganic MBBs assemble through (b) the formation of triple bridges. (c) Each MBB connected to four others in a tetrahedral fashion. (d) Tetrahedral MBBs oriented in eclipsed fashion, with (e) a slight tilt characteristic of zeolites, allowing the overall formation of $(f)$ the sodalite type cage.

161 encompasses microporous cages with a diameter of $\approx 6 \AA$, 162 resulting from the cantellation strategy, and delimited by the 163 three ligands bridging adjacent clusters (Figure S19a). Each 164 unit cell contains 3672 atoms, ca. 13.3 and 102 times more 165 than the unit cells of ZIF-8 (276 atoms) and the sodalite 166 zeolite (36 atoms), respectively.

167 From a purely geometrical view, the assembly of eclipsed 168 tetrahedra through our cantellation strategy should give an 169 ideal $\mathrm{T}-\mathrm{X}-\mathrm{T}$ angle of $180^{\circ}$ between the tetrahedral MBBs. 170 There is however no net allowing such perfect orientation of 171 eclipsed tetrahedra. Therefore, $\mathbf{m t n}$ or $\mathbf{m g z}-\mathbf{x}-\mathbf{d}$ nets, with T$172 \mathrm{X}-\mathrm{T}$ angles of 175 and $174.5^{\circ}$, respectively, could be the 173 expected topologies. ${ }^{34}$ Interestingly, the sod net is favored, 174 with an experimental $\mathrm{T}-\mathrm{X}-\mathrm{T}$ angle of $173.5^{\circ}$ (Figure S18c). 175 This can be explained by its higher regularity; compared to the 176 trinodal $\mathbf{m t n}$ and heptanodal $\mathbf{m g z}-\mathbf{x}-\mathbf{d}$ nets, the sod net is edge177 transitive and uninodal.
Interestingly, Zr-sod-ZMOF-1 contains two crystallograph- 178 ically independent ligands, both present in each of the triple 179 bridge between the clusters. The experimental bending angles 180 of $2 x 112.2^{\circ}$ and $1 \times 127^{\circ}$ were far from the ideal $120^{\circ}$ expected 181 for this type of bending (Figure S18a). This observation led us 182 to conclude that thanks to the slight natural flexibility of the 183 ligands the semiregular sod topology is ruling the MOF 184 assembly over the more ideal but less regular tetrahedra 185 stacking of $\mathbf{m t n}$ or $\mathbf{m g z}-\mathbf{x}-\mathbf{d}$. 186

The porosity of Zr-sod-ZMOF-1 has been examined by an 187 argon sorption experiment, exhibiting a type-IV isotherm, 188 characteristic of a mesoporous material (Figure 3a). The $189 \mathrm{f} 3$ apparent Brunauer-Emmett-Teller (BET) area was estimated 190 to be $1565 \mathrm{~m}^{2} \cdot \mathrm{g}^{-1}$. The total pore volume was calculated to be 191 $1.98 \mathrm{~cm}^{3} \cdot \mathrm{g}^{-1}$, in agreement with the theoretical value $\left(2.4 \mathrm{~cm}^{3} \cdot 192\right.$ $\left.\mathrm{g}^{-1}\right)$. This experimental pore volume is the highest for sod- 193 ZMOFs and even for ZMOFs based on 4-coordinated 194 tetrahedral MBBs (Figure 3c). ${ }^{35}$ The pore size distribution 195 

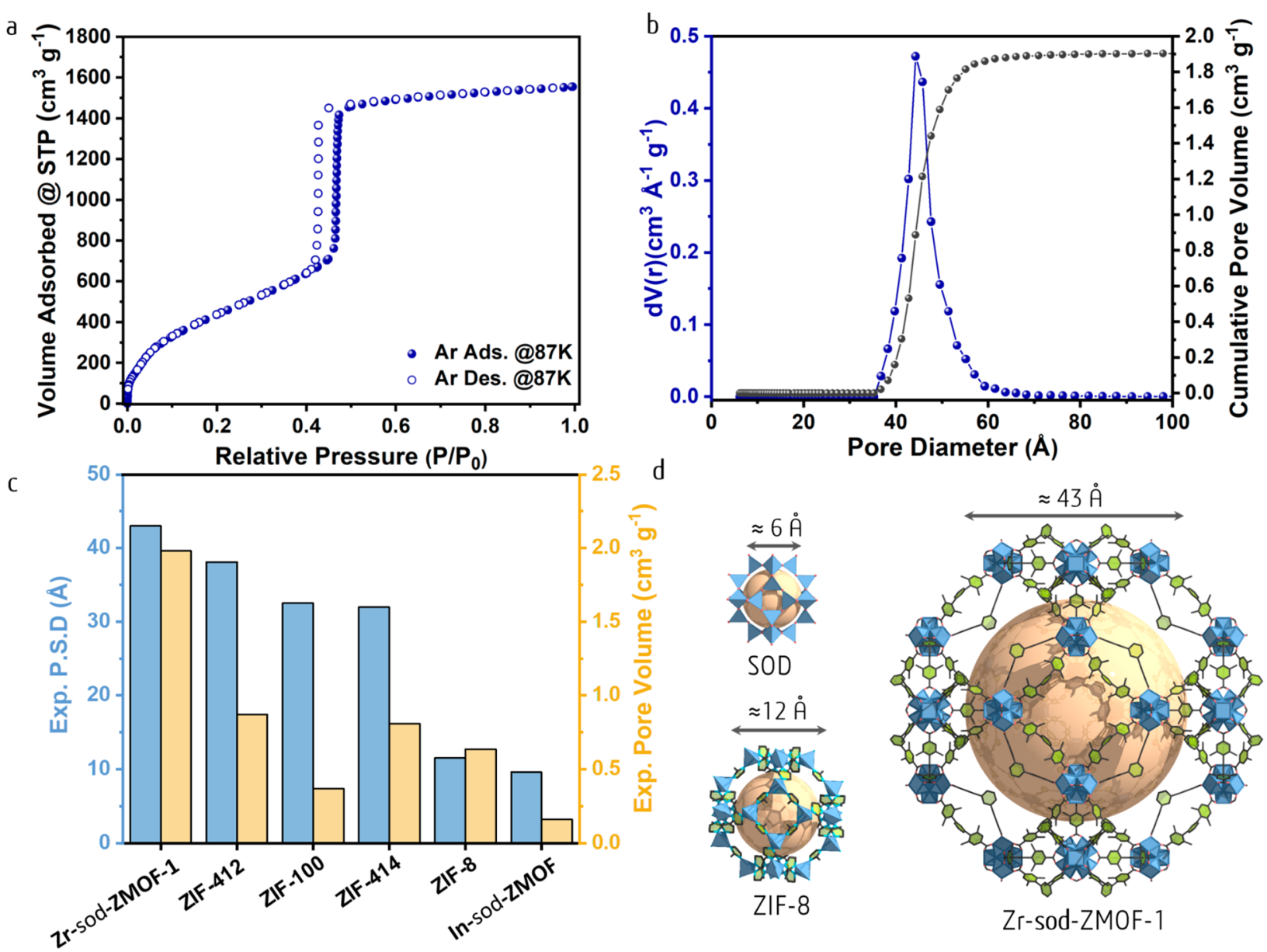

Figure 3. (a) Ar adsorption-desorption isotherm. (b) Corresponding PSD and (c) Experimental pore volume and PSD for Zr-sod-ZMOF-1 as compared to highest reported values from frameworks based on tetrahedral nodes or with sod topology. (d) Cage size for SOD structure, ZIF-8, and Zr-sod-ZMOF-1.
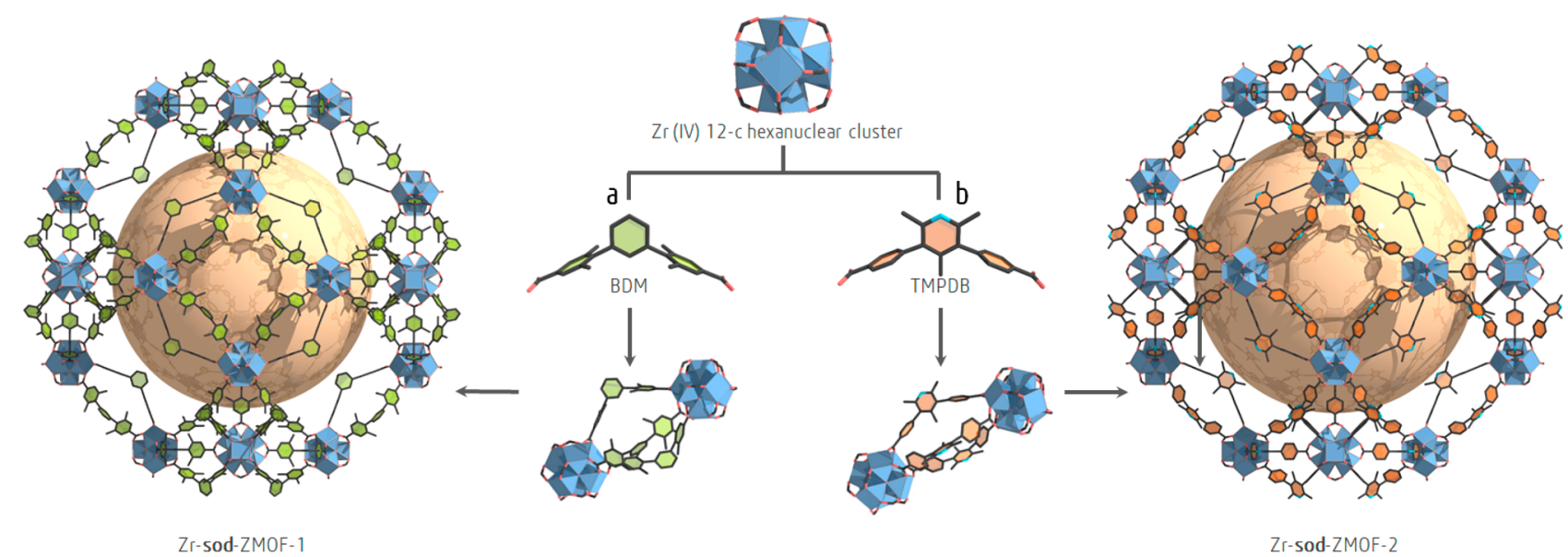

Zr-sod-ZMOF-2

Figure 4. Relocation of the steric hindrance from (a) the arms of the ligands ( $\mathrm{Zr}$-sod-ZMOF-1) to (b) the central core allows the formation of an isoreticular analogue, Zr-sod-ZMOF-2.

196 (PSD) shows a sharp pore size at $43 \AA$, in agreement with the 197 crystallographic data. Since the primary pore system in Zr-sod${ }_{198}$ ZMOF-1 is mesoporous, the model deployed could not assess 199 the contribution from the micropores. The micropores 200 between the clusters generated by the cantellation with 3 201 bent ligands can be relatively estimated by using only low202 pressure data points (up to $P / P_{0}=0.03$ ), asserting the presence of micropores around $6.2 \AA$ and their contribution to 203 the overall porosity (Figure S24c).

In order to assess the strength of our cantellation design 205 approach, we intended to prepare isoreticular Zr-sod-ZMOF 206 (Figure 4), and a novel ligand was designed. 4,4'-(2,4,6- $207 \mathrm{f4}$ Trimethylpyridine)dibenzoic acid (TMPDB, L2) has the same 208 angular characteristics as L1; however, its steric hindrance is 209 now located in the central part of the ligand, on a pyridine ring. 210 
211 As expected, the use of ligand L2 allowed to obtain an 212 isoreticular analogue to Zr-sod-ZMOF-1 (Tables S1 and S2). 213 The phase purity was further confirmed by powder X-ray 214 diffraction (PXRD, Figure S17). Due to the similarities 215 between L1 and L2, Zr-sod-ZMOF-2 exhibits the same type 216 of cages: the mesoporous cage, having a diameter of about 43.5 $217 \AA$, and a confined cage between the triple ligands of 4-6 218 with two windows, a square of $12 \AA$ and a hexagon of $22 \AA$ 219 (Figure S19b). Similar to Zr-sod-ZMOF-1, there are two 220 crystallographically independent ligands in the Zr-sod-ZMOF$2212\left(2 \times 118^{\circ}\right.$ and $\left.1 \times 130.4^{\circ}\right)$, and the tetrahedral units show a $222 \mathrm{~T}-\mathrm{X}-\mathrm{T}$ angle of $173^{\circ}$ (Figure S18a).

223 In summary, we developed a cantellation design approach 224 that afforded the deployment of the 12-c Zr-based MBB as a 225 tetrahedral secondary building unit. Locked in an eclipsed 226 conformation through triple bridging by the ligands, the 227 stacking of the MBBs prevents the formation of inter228 penetrated MOFs with dia topology while allowing the 229 formation of zeolitic-nets. This strategy permitted the 230 discovery of two isoreticular mesoporous Zr-sod-ZMOFs, the 231 first examples of Zr-MOFs with zeolitic topology. Zr-sod232 ZMOF-1 has the highest experimental pore volume reported 233 for any sodalite type material so far $(\approx 3.5$ times higher than 234 ZIF-8), as well as for any zeolitic material based on tetrahedral 235 MBBs. Finally, our cantellation strategy opens new oppor236 tunities for the deliberate use of highly coordinated clusters as 237 secondary building units with lower connectivity through 238 multiple bridging.

\section{ASSOCIATED CONTENT}

\section{SI Supporting Information}

241 The Supporting Information is available free of charge at 242 https://pubs.acs.org/doi/10.1021/jacs.0c10007.

243 Synthesis, PXRD, TGA, and additional sorption data 244 (PDF)

245 Crystallographic data for 1-periodic phase, Zr-sod246 ZMOF-1, and Zr-sod-ZMOF-2 (CIF, CIF, and CIF)

\section{AUTHOR INFORMATION} 248 Corresponding Author

249 Mohamed Eddaoudi - Functional Materials Design, 250 Discovery and Development Research Group (FMD3), 251 Advanced Membranes and Porous Materials Center 252 (AMPM), Division of Physical Sciences and Engineering 253 (PSE), King Abdullah University of Science and Technology 254 (KAUST), Thuwal 23955-6900, Saudi Arabia;

255 ○ orcid.org/0000-0003-1916-9837;

256 Email: mohamed.eddaoudi@kaust.edu.sa

\section{Authors}

258 Norah Alsadun - Functional Materials Design, Discovery and 259 Development Research Group (FMD3), Advanced of Physical Sciences and Engineering (PSE), King Abdullah University of Science and Technology (KAUST), Thuwal 23955-6900, Saudi Arabia; Department of Chemistry, College of Science, King Faisal University (KFU), Al-Ahsa 31982-400, Saudi Arabia

Georges Mouchaham - Functional Materials Design, Discovery and Development Research Group (FMD3), Advanced Membranes and Porous Materials Center (AMPM), Division of Physical Sciences and Engineering
(PSE), King Abdullah University of Science and Technology 270 (KAUST), Thuwal 23955-6900, Saudi Arabia 271

Vincent Guillerm - Functional Materials Design, Discovery 272 and Development Research Group (FMD3), Advanced 273 Membranes and Porous Materials Center (AMPM), Division 274 of Physical Sciences and Engineering (PSE), King Abdullah 275 University of Science and Technology (KAUST), Thuwal 276 23955-6900, Saudi Arabia; 이이.org/0000-0003-3460- 277 $223 \mathrm{X}$

Justyna Czaban-Jóźwiak - Functional Materials Design, 279 Discovery and Development Research Group (FMD3), 280 Advanced Membranes and Porous Materials Center 281 (AMPM), Division of Physical Sciences and Engineering 282 (PSE), King Abdullah University of Science and Technology 283 (KAUST), Thuwal 23955-6900, Saudi Arabia 284

Aleksander Shkurenko - Functional Materials Design, 285 Discovery and Development Research Group (FMD3), 286 Advanced Membranes and Porous Materials Center 287 (AMPM), Division of Physical Sciences and Engineering 288 (PSE), King Abdullah University of Science and Technology 289 (KAUST), Thuwal 23955-6900, Saudi Arabia; 290 (1) orcid.org/0000-0001-7136-2277 291

Hao Jiang - Functional Materials Design, Discovery and 292 Development Research Group (FMD3), Advanced 293 Membranes and Porous Materials Center (AMPM), Division 294 of Physical Sciences and Engineering (PSE), King Abdullah 295 University of Science and Technology (KAUST), Thuwal 296 23955-6900, Saudi Arabia; (1) orcid.org/0000-0002-1234- 297 $624 \mathrm{X}$

298

Prashant M. Bhatt - Functional Materials Design, Discovery 299 and Development Research Group (FMD3), Advanced 300 Membranes and Porous Materials Center (AMPM), Division 301 of Physical Sciences and Engineering (PSE), King Abdullah 302 University of Science and Technology (KAUST), Thuwal 303 23955-6900, Saudi Arabia

Prakash Parvatkar - Functional Materials Design, Discovery 305 and Development Research Group (FMD3), Advanced 306 Membranes and Porous Materials Center (AMPM), Division 307 of Physical Sciences and Engineering (PSE), King Abdullah 308 University of Science and Technology (KAUST), Thuwal 309 23955-6900, Saudi Arabia

Complete contact information is available at:

https://pubs.acs.org/10.1021/jacs.0c10007

Author Contributions

312

${ }^{\S}$ N.A. and G.M. contributed equally to this work. 314

Notes

The authors declare no competing financial interest. 316

\section{ACKNOWLEDGMENTS}

317

Research reported in this publication was supported by the 318 King Abdullah University of Science and Technology 319 (KAUST).

\section{REFERENCES}

321

(1) (a) Eddaoudi, M.; Kim, J.; Rosi, N.; Vodak, D.; Wachter, J.; 322 Keeffe, M.; Yaghi, O. M. Systematic Design of Pore Size and 323 Functionality in Isoreticular MOFs and Their Application in Methane 324 Storage. Science 2002, 295 (5554), 469. (b) Kitagawa, S.; Kitaura, R.; 325 Noro, S.-i. Functional Porous Coordination Polymers. Angew. Chem., 326 Int. Ed. 2004, 43 (18), 2334-2375. (c) Eddaoudi, M.; Moler, D. B.; 327 Li, H. L.; Chen, B. L.; Reineke, T. M.; O'Keeffe, M.; Yaghi, O. M. 328 Modular chemistry: Secondary building units as a basis for the design 329 
330 of highly porous and robust metal-organic carboxylate frameworks. 331 Acc. Chem. Res. 2001, 34 (4), 319-330.

332 (2) Horcajada, P.; Gref, R.; Baati, T.; Allan, P. K.; Maurin, G.; 333 Couvreur, P.; Férey, G.; Morris, R. E.; Serre, C. Metal-Organic 334 Frameworks in Biomedicine. Chem. Rev. 2012, 112 (2), 1232-1268. 335 (3) Adil, K.; Belmabkhout, Y.; Pillai, R. S.; Cadiau, A.; Bhatt, P. M.; 336 Assen, A. H.; Maurin, G.; Eddaoudi, M. Gas/vapour separation using 337 ultra-microporous metal-organic frameworks: insights into the 338 structure/separation relationship. Chem. Soc. Rev. 2017, 46 (11), $3393402-3430$.

340 (4) Kreno, L. E.; Leong, K.; Farha, O. K.; Allendorf, M.; Van Duyne, 341 R. P.; Hupp, J. T. Metal-Organic Framework Materials as Chemical 342 Sensors. Chem. Rev. 2012, 112 (2), 1105-1125.

343 (5) Moghadam, P. Z.; Li, A.; Wiggin, S. B.; Tao, A.; Maloney, A. G. 344 P.; Wood, P. A.; Ward, S. C.; Fairen-Jimenez, D. Development of a 345 Cambridge Structural Database Subset: A Collection of Metal346 Organic Frameworks for Past, Present, and Future. Chem. Mater. 347 2017, 29 (7), 2618-2625.

348 (6) (a) Yaghi, O. M.; O'Keeffe, M.; Ockwig, N. W.; Chae, H. K.; 349 Eddaoudi, M.; Kim, J. Reticular synthesis and the design of new 350 materials. Nature 2003, 423, 705. (b) O’Keeffe, M.; Yaghi, O. M. 351 Deconstructing the Crystal Structures of Metal-Organic Frameworks 352 and Related Materials into Their Underlying Nets. Chem. Rev. 2012, 353112 (2), 675-702. (c) O'Keeffe, M.; Peskov, M. A.; Ramsden, S. J.; 354 Yaghi, O. M. The Reticular Chemistry Structure Resource (RCSR) 355 Database of, and Symbols for, Crystal Nets. Acc. Chem. Res. 2008, 41 356 (12), 1782-1789.

357 (7) Chen, Z.; Hanna, S. L.; Redfern, L. R.; Alezi, D.; Islamoglu, T.; 358 Farha, O. K. Reticular chemistry in the rational synthesis of functional 359 zirconium cluster-based MOFs. Coord. Chem. Rev. 2019, 386, 32-49. 360 (8) Chen, Z.; Jiang, H.; Li, M.; O'Keeffe, M.; Eddaoudi, M. Reticular 361 Chemistry 3.2: Typical Minimal Edge-Transitive Derived and Related 362 Nets for the Design and Synthesis of Metal-Organic Frameworks. 363 Chem. Rev. 2020, 120, 8039.

364 (9) Guillerm, V.; Maspoch, D. Geometry mismatch and reticular 365 chemistry: strategies to assemble metal-organic frameworks with non366 default topologies. J. Am. Chem. Soc. 2019, 141 (42), 16517-16538. 367 (10) Chun, H.; Dybtsev, D. N.; Kim, H.; Kim, K. Synthesis, X-ray 368 Crystal Structures, and Gas Sorption Properties of Pillared Square 369 Grid Nets Based on Paddle-Wheel Motifs: Implications for Hydrogen 370 Storage in Porous Materials. Chem. - Eur. J. 2005, 11 (12), 35213713529.

372 (11) Eubank, J. F.; Mouttaki, H.; Cairns, A. J.; Belmabkhout, Y.; 373 Wojtas, Ł.; Luebke, R.; Alkordi, M.; Eddaoudi, M. The Quest for 374 Modular Nanocages: tbo-MOF as an Archetype for Mutual 375 Substitution, Functionalization, and Expansion of Quadrangular Pillar 376 Building Blocks. J. Am. Chem. Soc. 2011, 133 (36), 14204-14207.

377 (12) Nouar, F.; Eubank, J. F.; Bousquet, T.; Wojtas, Ł.; Zaworotko, 378 M. J.; Eddaoudi, M. Supermolecular building blocks (SBBs) for the 379 design and synthesis of highly porous metal-organic frameworks. J. $380 \mathrm{Am}$. Chem. Soc. 2008, 130 (6), 1833-1834.

381 (13) Eddaoudi, M.; Sava, D. F.; Eubank, J. F.; Adil, K.; Guillerm, V. 382 Zeolite-like metal-organic frameworks (ZMOFs): design, synthesis, 383 and properties. Chem. Soc. Rev. 2015, 44 (1), 228-249.

384 (14) (a) Liu, Y.; Kravtsov, V. C.; Larsen, R.; Eddaoudi, M. Molecular 385 building blocks approach to the assembly of zeolite-like metal386 organic frameworks (ZMOFs) with extra-large cavities. Chem. 387 Commun. 2006, 14, 1488-1490. (b) Huang, X.-C.; Lin, Y.-Y.; 388 Zhang, J.-P.; Chen, X.-M. Ligand-Directed Strategy for Zeolite-Type 389 Metal-Organic Frameworks: Zinc(II) Imidazolates with Unusual 390 Zeolitic Topologies. Angew. Chem., Int. Ed. 2006, 45 (10), 1557391 1559. (c) Wang, B.; Côté, A. P.; Furukawa, H.; O’Keeffe, M.; Yaghi, 392 O. M. Colossal cages in zeolitic imidazolate frameworks as selective 393 carbon dioxide reservoirs. Nature 2008, 453 (7192), 207-211. 394 (d) Hayashi, H.; Côté, A. P.; Furukawa, H.; O’Keeffe, M.; Yaghi, O. 395 M. Zeolite A imidazolate frameworks. Nat. Mater. 2007, 6 (7), 501396506.

397 (15) Liu, Y.; Kravtsov, V.; Eddaoudi, M. Template-directed assembly 398 of zeolite-like metal-organic frameworks (ZMOFs): a usf-ZMOF with an unprecedented zeolite topology. Angew. Chem., Int. Ed. 2008, 47399 (44), 8446-9.

400

(16) Férey, G.; Mellot-Draznieks, C.; Serre, C.; Millange, F.; Dutour, 401 J.; Surblé, S.; Margiolaki, I. A chromium terephthalate-based solid 402 with unusually large pore volumes and surface area. Science 2005, 309403 (5743), 2040-2042.

(17) Férey, G.; Serre, C.; Mellot-Draznieks, C.; Millange, F.; Surblé, 405 S.; Dutour, J.; Margiolaki, I. A hybrid solid with giant pores prepared 406 by a combination of targeted chemistry, simulation, and powder 407 diffraction. Angew. Chem., Int. Ed. 2004, 43 (46), 6296-6301. 408

(18) Horcajada, P.; Chevreau, H.; Heurtaux, D.; Benyettou, F.; 409 Salles, F.; Devic, T.; Garcia-Marquez, A.; Yu, C.; Lavrard, H.; Dutson, 410 C. L.; Magnier, E.; Maurin, G.; Elkaïm, E.; Serre, C. Extended and 411 functionalized porous iron(iii) tri- or dicarboxylates with MIL-100/ 412 101 topologies. Chem. Commun. 2014, 50 (52), 6872-6874.

413

(19) (a) Guillerm, V.; Kim, D.; Eubank, J. F.; Luebke, R.; Liu, X.; 414 Adil, K.; Lah, M. S.; Eddaoudi, M. A supermolecular building 415 approach for the design and construction of metal-organic 416 frameworks. Chem. Soc. Rev. 2014, 43 (16), 6141-6172. (b) Kal- 417 mutzki, M. J.; Hanikel, N.; Yaghi, O. M. Secondary building units as 418 the turning point in the development of the reticular chemistry of 419 MOFs. Science Adv. 2018, 4 (10), No. eaat9180. (c) Guillerm, V.; 420 Grancha, T.; Imaz, I.; Juanhuix, J.; Maspoch, D. Zigzag Ligands for 421 Transversal Design in Reticular Chemistry: Unveiling New Structural 422 Opportunities for Metal-Organic Frameworks. J. Am. Chem. Soc. 423 2018, 140 (32), 10153-10157.

(20) Zaworotko, M. J. Crystal engineering of diamondoid networks. 425 Chem. Soc. Rev. 1994, 23 (4), 283-288.

(21) (a) Park, K. S.; Ni, Z.; Côté, A. P.; Choi, J. Y.; Huang, R.; 427 Uribe-Romo, F. J.; Chae, H. K.; O’Keeffe, M.; Yaghi, O. M. 428 Exceptional chemical and thermal stability of zeolitic imidazolate 429 frameworks. Proc. Natl. Acad. Sci. U. S. A. 2006, 103 (27), 10186- 430 10191. (b) Zhang, J.; Wu, T.; Zhou, C.; Chen, S.; Feng, P.; Bu, X. 431 Zeolitic Boron Imidazolate Frameworks. Angew. Chem., Int. Ed. 2009, 432 48 (14), 2542-2545. (c) Huang, X.; Zhang, J.; Chen, X. [Zn(bim) 2$] \cdot 433$ $\left(\mathrm{H}_{2} \mathrm{O}\right)$ 1.67: A metal-organic open-framework with sodalite topology. 434 Chin. Sci. Bull. 2003, 48 (15), 1531-1534. (d) Noh, K.; Lee, J.; Kim, 435 J. Compositions and Structures of Zeolitic Imidazolate Frameworks. 436 Isr. J. Chem. 2018, 58 (9-10), 1075-1088.

(22) Delgado Friedrichs, O.; O'Keeffe, M.; Yaghi, O. M. Three- 438 periodic nets and tilings: regular and quasiregular nets. Acta 439 Crystallogr., Sect. A: Found. Crystallogr. 2003, 59 (1), 22-27. 440

(23) Cavka, J. H.; Jakobsen, S.; Olsbye, U.; Guillou, N.; Lamberti, 441 C.; Bordiga, S.; Lillerud, K. P. A new zirconium inorganic building 442 brick forming metal organic frameworks with exceptional stability. J. 443 Am. Chem. Soc. 2008, 130 (42), 13850-13851.

(24) Perfecto-Irigaray, M.; Beobide, G.; Castillo, O.; da Silva, I.; 445 García-Lojo, D.; Luque, A.; Mendia, A.; Pérez-Yáñez, S. 446 $\left.\left[\mathrm{Zr}_{6} \mathrm{O}_{4}(\mathrm{OH})_{4} \text { (benzene-1,4-dicarboxylato }\right)_{6}\right]_{\mathrm{n}}$ : a hexagonal polymorph 447 of UiO-66. Chem. Commun. 2019, 55 (42), 5954-5957. 448

(25) (a) Guillerm, V.; Gross, S.; Serre, C.; Devic, T.; Bauer, M.; 449 Férey, G. A zirconium methacrylate oxocluster as precursor for the 450 low-temperature synthesis of porous zirconium(IV) dicarboxylates. 451 Chem. Commun. 2010, 46 (5), 767-769. (b) Schate, A.; Roy, P.; 452 Godt, A.; Lippke, J.; Waltz, F.; Wiebcke, M.; Behrens, P. Modulated 453 Synthesis of Zr-Based Metal-Organic Frameworks: From Nano to 454 Single Crystals. Chem. - Eur. J. 2011, 17 (24), 6643-6651. 455 (c) Winarta, J.; Shan, B.; McIntyre, S. M.; Ye, L.; Wang, C.; Liu, J.; 456 $\mathrm{Mu}, \mathrm{B}$. A Decade of UiO-66 Research: A Historic Review of Dynamic 457 Structure, Synthesis Mechanisms, and Characterization Techniques of 458 an Archetypal Metal-Organic Framework. Cryst. Growth Des. 2020, 459 20 (2), 1347-1362.

(26) Bon, V.; Senkovska, I.; Baburin, I. A.; Kaskel, S. Zr- and Hf- 461 Based Metal-Organic Frameworks: Tracking Down the Poly- 462 morphism. Cryst. Growth Des. 2013, 13 (3), 1231-1237. 463

(27) Yuan, S.; Lu, W.; Chen, Y.-P.; Zhang, Q.; Liu, T.-F.; Feng, D.; 464 Wang, X.; Qin, J.; Zhou, H.-C. Sequential Linker Installation: Precise 465 Placement of Functional Groups in Multivariate Metal-Organic 466 Frameworks. J. Am. Chem. Soc. 2015, 137 (9), 3177-3180. 
468 (28) Wang, B.; Huang, H.; Lv, X.-L.; Xie, Y.; Li, M.; Li, J.-R. Tuning $469 \mathrm{CO}_{2}$ Selective Adsorption over $\mathrm{N}_{2}$ and $\mathrm{CH}_{4}$ in UiO-67 Analogues 470 through Ligand Functionalization. Inorg. Chem. 2014, 53 (17), 92544719259 .

472 (29) Bon, V.; Senkovskyy, V.; Senkovska, I.; Kaskel, S. Zr(iv) and $473 \mathrm{Hf}(\mathrm{iv})$ based metal-organic frameworks with reo-topology. Chem. 474 Commun. 2012, 48 (67), 8407-8409.

475 (30) Furukawa, H.; Gándara, F.; Zhang, Y.-B.; Jiang, J.; Queen, W. 476 L.; Hudson, M. R.; Yaghi, O. M. Water Adsorption in Porous Metal477 Organic Frameworks and Related Materials. J. Am. Chem. Soc. 2014, 478136 (11), 4369-4381.

479 (31) Drache, F.; Bon, V.; Senkovska, I.; Getzschmann, J.; Kaskel, S. 480 The modulator driven polymorphism of $\mathrm{Zr}(\mathrm{IV})$ based metal-organic 481 frameworks. Philos. Trans. R. Soc., A 2017, 375 (2084), 20160027.

482 (32) Dreischarf, A. C.; Lammert, M.; Stock, N.; Reinsch, H. Green 483 Synthesis of Zr-CAU-28: Structure and Properties of the First $\mathrm{Zr}$ 484 MOF Based on 2,5-Furandicarboxylic Acid. Inorg. Chem. 2017, 56 485 (4), 2270-2277.

486 (33) Xie, L.-H.; Liu, X.-M.; He, T.; Li, J.-R. Metal-Organic 487 Frameworks for the Capture of Trace Aromatic Volatile Organic 488 Compounds. Chem. 2018, 4 (8), 1911-1927.

489 (34) O'Keeffe, M. Tetrahedral frameworks $\mathrm{TX}_{2}$ with $\mathrm{T}-\mathrm{X}-\mathrm{T}$ 490 angle $=180^{\circ}$ : Rationalization of the structures of MOF-500 and of 491 MIL-100 and MIL-101. Mater. Res. Bull. 2006, 41 (5), 911-915.

492 (35) Yang, J.; Zhang, Y.-B.; Liu, Q.; Trickett, C. A.; Gutiérrez493 Puebla, E.; Monge, M. Á.; Cong, H.; Aldossary, A.; Deng, H.; Yaghi, 494 O. M. Principles of Designing Extra-Large Pore Openings and Cages 495 in Zeolitic Imidazolate Frameworks. J. Am. Chem. Soc. 2017, 139 (18), $4966448-6455$. 